use, chronic pulmonary disease, diabetes, cancer and cardiovascular disease were all associated with an increased mortality.

Disclosure of Interests: : None declared

DOI: 10.1136/annrheumdis-2020-eular.1203

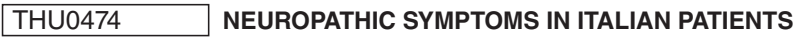 WITH FIBROMYALGIA: RESULTS FROM A NATIONAL ON-LINE SURVEY}

A. Viceconti ${ }^{1}$, T. Geri ${ }^{1}$, S. De Luca ${ }^{1}$, F. Maselli ${ }^{1}$, A. Sulli ${ }^{2}$, M. Testa ${ }^{1}$,

G. Rossettini'. ' University of Genoa, Department of Neuroscience, Rehabilitation, Ophthalmology, Genetics, Maternal and Child Health, Savona, Italy; ${ }^{2}$ University of Genoa, Research Laboratory and Academic Division of Clinical Rheumatology, Department of Internal Medicine, IRCCS San Martino AOU, Genoa, Italy

Background: Fibromyalgia (FM) is the second most frequent disorder in rheumatic patients. Other than widespread pain, fatigue, sleep disturbance and cognitive impairments, patients complain also symptoms of suspected neuropathic origin, like burning pain, thermal sensitive skin, hyperalgesia, pins and needles sensations. Recent studies highlighted the presence of small- fibers pathology (SFP) and/or large-nerve fibers involvement in about $50 \%$ of FM patients, which could be the cause of neuropathic pain.

Objectives: The aim of the study was to investigate the prevalence of neuropathic pain and symptoms indicative for the presence of SFP in Italian FM patients, studying the association with clinical variables.

Methods: An on-line survey was designed according to the Checklist for Reporting Results of Internet E-Surveys guidelines (CHERRIES) and Strengthening the Reporting of Observational Studies in Epidemiology (STROBE). The on-line Survey Monkey® platform was adopted to collect data. We calculated a-priori minimum number of 800 responders.

We administered the survey by involving 7 FM patients' associations distributed nationwide between July and September 2019. We explored demographic and clinical variables including pain and stiffness intensity, symptoms duration, and counting of painful sites. Neuropathic Pain Symptoms Inventory (NPSI) and Fibromyalgia Impact Questionnaire (FIQ) were administered. To study the presence of symptoms indicative of potential SFP we asked for the presence of 8 signs and symptoms reported in literature as characteristics of SFPs. Two groups of FM patients were considered: those positive (FM+) to the Fibromyalgia Research Criteria (FRC) (Wolfe et al., 2011), and those complaining typical FM symptoms but not fulfilling the FRC (FM-).

Results: The survey was correctly completed by $76 \%$ of participants (892/1173). A total sample of 854 patients (749 in FM+ and 105 in FM-) was analyzed after the exclusion of subjects with major comorbidities. The mean NPSI score was significantly higher in $\mathrm{FM}+(56.3 / 100)$ respect to $\mathrm{FM}-(34.2 / 100)$. NPSI score was $\geq 50 / 100$ in $62.4 \%$ of $F M+$ patients and in $21.0 \%$ of FM-. More than 3 symptoms indicative for SFP were found in $51 \%$ of FM+ patients and in $15.2 \%$ of FM-. Dry eyes/mouth, allodynia and changed pattern of sweating on body were the 3 most frequently reported symptoms. We found statistically significant strong associations $(p<0.001)$ for the NPSI score with disability $(r h o=0.53)$ and pain $(r h o=0.63)$, and a moderate correlation with stiffness levels $(r h o=0.45)$ and counting of painful sites $(r h o=0.33)$. Symptoms indicative for SFP were significantly correlated $(p<0.001)$ with all clinical variables with low grade of association (Cramer's $V$ or $r h o<0.30)$. Although the higher prevalence of neuropathic pain and symptoms potentially indicative for SFPs, electromyography and electroneurography were performed in $40 \%-44 \%$ of cases, and skin biopsy in $1 \%-2 \%$ of the sample, as well as the assumption of gabapentinoids ( $12.6 \%$ in $\mathrm{FM}+$ and $18 \%$ in FM-).

Conclusion: This findings highlight the importance of neuropathic pain symptoms identification, since we found a high prevalence and a strong correlation with clinical variables in our cohort of FM patients. The assessment of the neuropathic dimension of pain through self-administered questionnaire should be part of the routine clinical practice.

References:

[1] Wolfe, F., et al., 2011. Fibromyalgia Criteria and Severity Scales for Clinical and Epidemiological Studies: A Modification of the ACR Preliminary Diagnostic Criteria for Fibromyalgia. The Journal of Rheumatology 38, 1113-1122.
Figu re 1. Enrollment process flow-ch art

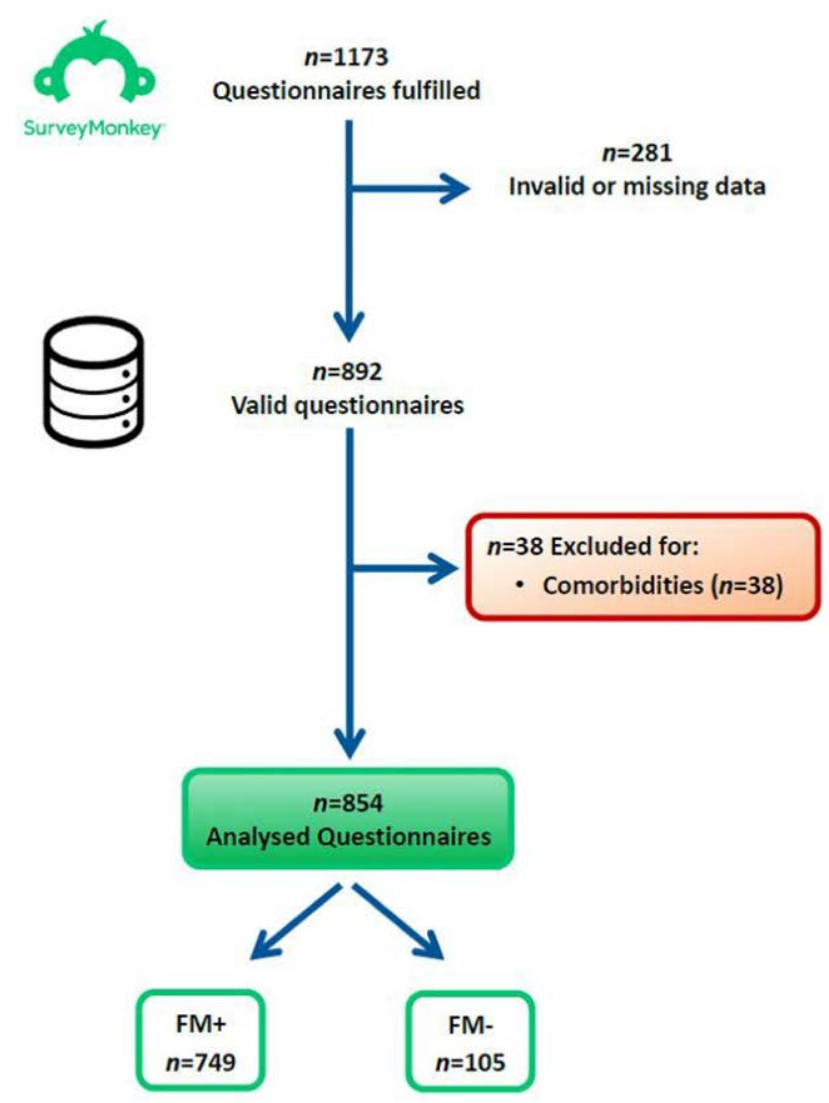

Disclosure of Interests: : Antonello Viceconti: None declared, Tommaso Geri: None declared, Simone De Luca: None declared, Filippo Maselli: None declared, Alberto Sulli Grant/research support from: Laboratori Baldacci, Marco Testa: None declared, Giacomo Rossettini: None declared

DOI: 10.1136/annrheumdis-2020-eular.3547

\section{THU0475 THE EFFICACY OF ORAL GLUCOCORTICOSTEROIDS FOR PAIN IN RHEUMATOID ARTHRITIS: A PRELIMINARY REPORT OF A META-ANALYSIS}

D. Mcwilliams $s^{1,2,3}$, D. Thankaraj ${ }^{1,3}$, R. Morgan ${ }^{4}$, J. Jones-Diette ${ }^{1,3}$, D. Walsh ${ }^{1,2,3,5}$. ${ }^{1}$ University of Nottingham, Pain Centre Versus Arthritis, Nottingham, United Kingdom; ${ }^{2}$ University of Nottingham, NIHR Nottingham Biomedical Research Centre, Nottingham, United Kingdom; ${ }^{3}$ University of Nottingham, Division of ROD, Nottingham, United Kingdom; ${ }^{4}$ Nottingham University Hospitals NHS Trust, Surgery, Nottingham, United Kingdom; ${ }^{5}$ Sherwood Forest Hospitals NHS Foundation Trust, Rheumatology, Mansfield, United Kingdom

Background: Glucocorticosteroids (GCs) are used to provide rapid relief of symptoms in people with active RA. Their use is recommended by most RA management guidelines and systematic reviews, although the magnitude of their benefit above placebo is uncertain. Persistent pain remains a problem in RA, even despite optimal immunomodulatory management. Systemic GC use may be associated with important adverse events.

Objectives: To quantify the specific effects of oral GCs for RA pain.

Methods: A systematic literature review was performed for RCTs using GCs in RA compared to inactive treatment. Trials were included whether or not participants received DMARD treatments, so long as a specific effect could be assigned to GCs. Medline, Embase and Cochrane databases were searched until November 2019 and 2 reviewers independently assessed titles, abstracts and full texts. Data for pain were synthesized in a meta-analysis. This study is part of a wider review (PROSPERO CRD42019111562).

For subgroup analyses, follow up time points of 0-3 months, $>3-6$ months and $>6$ months were selected to address duration of effect. Individual studies could contribute to each of the 3 follow up subgroups. 
Meta-analysis was performed on standardized mean differences (SMDs, bodily pain data) and mean differences (MDs, 100mm VASpain only) of change from baseline (sd), using the Meta and Metafor packages in R. Heterogeneity was quantified using $\mathrm{I}^{2}$ and tau statistics. Bias was assessed with a funnel plot and Eggers test.

Results: 15983 papers, 470 abstracts and 152 full texts were assessed. Pain data from 12 RCTs were suitable for the meta-analyses. The most common pain metric was the $100 \mathrm{~mm}$ VASpain ( 9 trials).

Study populations ranged from $n=12$ to $n=350$ participants, $50 \%$ to $71 \%$ were female with mean ages from 43 to 66 years. Baseline scores for VASpain ranged from to 34 to $66 \mathrm{~mm}$. Means were reported for DAS28 (from 4.9 to 5.8), ESR (25 to $60 \mathrm{~mm}$ ) and CRP (5 to $27 \mathrm{mg} / \mathrm{L}$ ).

Data synthesis at the reported primary time point/end point showed a statistically significant reduction in bodily pain in participants treated with GCs; SMD $=-0.36$ (10 studies, 1377 participants, $95 \% \mathrm{Cl},-0.59$ to $-0.14, \mathrm{p}=0.002$ ) with significant heterogeneity $\left(\mathrm{I}^{2}=66 \%\right.$, tau $\left.=0.27, \mathrm{p}<0.01\right)$. The Funnel plot suggested asymmetry, favouring GCs (Eggers $\mathrm{p}=0.007$ ).

Subgroup analyses were used to investigate the time course of specific effects on pain. Efficacy displayed time-related decreases after initiation. From 0-3 months $\mathrm{SMD}=-0.56\left(95 \% \mathrm{Cl},-0.76\right.$ to $-0.36, \mathrm{p}<0.001,9$ studies, 936 participants, $\mathrm{I}^{2}=$ $43 \%$, Eggers $\mathrm{p}=0.002)$. Efficacy was lower at $>3-6$ month $(\mathrm{SMD}=-0.32,95 \% \mathrm{Cl}$ -0.52 to $-0.11, p=0.002,3$ studies, 382 participants, $\mathrm{I}^{2}=0 \%$, Eggers $\mathrm{p}=0.75$ ) and further reduced at $>6$ months $(\mathrm{SMD}=-0.07,95 \% \mathrm{Cl},-0.23$ to $0.08, \mathrm{p}=0.357,4$ studies, 665 participants, $\mathrm{I}^{2}=7 \%$, Eggers $\mathrm{p}=0.43$ ).

For trial data collected during concomitant oral GC dosage, mean difference (MDs) in $100 \mathrm{~mm}$ VASpain was $-14 \mathrm{~mm}(95 \% \mathrm{Cl},-20 \mathrm{~mm}$ to $-9 \mathrm{~mm})$ greater improvement in GC than control in the 0-3 month period (8 studies, 1047 participants, $\left.I^{2}=70 \%\right)$. For later follow ups, MDs at $>3$ to 6 months were $-6 \mathrm{~mm}(95 \%$ $\mathrm{Cl},-11 \mathrm{~mm}$ to $-1 \mathrm{~mm}, 3$ studies, 537 participants), and $-1 \mathrm{~mm}(95 \% \mathrm{Cl},-6 \mathrm{~mm}$ to $4 \mathrm{~mm}, 3$ studies, 369 participants) at $>6$ months.

Conclusion: Oral GCs have efficacy for pain in RA but the mean effect is of borderline clinical importance, and greatest shortly after steroids are commenced. GCs were usually used alongside other treatments as part of a combination. Future research might determine who might gain most benefit from systemic GCs, and improve other treatments to reduce the burden of pain.

Acknowledgments: Dr Douglas Grindley for help devising search strategy Disclosure of Interests: : Daniel McWilliams Grant/research support from: Grant support from Pfizer Ltd, Divya Thankaraj: None declared, Rheinallt Morgan: None declared, Julie Jones-Diette: None declared, David Walsh Grant/ research support from: Grant support from Pfizer Ltd and Eli Lilly, Consultant of: Consultancy to Eli Lilly, Pfizer, Abbvie and GSK (all payments made to University of Nottingham). Consultancy to Love Productions(all payments made to the University of Nottingham).

DOI: 10.1136/annrheumdis-2020-eular.3710

\section{THURSDAY, 04 JUNE 2020}

\section{Back pain, mechanical musculoskeletal problems, local soft tissue disorders}

\section{THU0476 GLYCOSAMINOGLYCAN REMODELLING OF LUMBAR INTERVERTEBRAL DISCS IN ELITE ROWERS THROUGHOUT THEIR ANNUAL TRAINING CYCLE}

P. Sewerin ${ }^{1}$, D. Abrar ${ }^{2}$, M. Frenken², M. Schneider ${ }^{1}$, B. Ostendorf ${ }^{1}$, C. Schleich ${ }^{2}$. ${ }^{1}$ Heinrich Heine University, Department for Rheumatology, Duesseldorf, Germany; ${ }^{2}$ Heinrich-Heine University, Institute for Diagnostic and Interventional Radiology, Duesseldorf, Germany

Background: To assess the glycosaminoglycan (GAG) content of lumbar intervertebral discs (IVD) in elite rowers (ER) at different stages of their annual training cycle and compared to healthy volunteers (HV) using GAG chemical exchange saturation transfer (gagCEST).

Objectives: Does GAG content of IVDs differs between ER in different stages of the training cycle from $\mathrm{HV}$ ?

Methods: 205 lumbar IVD of $21 \mathrm{ER}(23 \pm 3$ years, 9 female, 11 male) and $25 \mathrm{HV}$ ( $27 \pm 2$ years, 13 female, 12 male) were prospectively examined with $3 T$ magnetic resonance imaging (MRI). Standard T2 weighted (T2w) sequences were used for morphological grading according to the Pfirrmann classification. GAG content of the nucleus pulposus (NP) and annulus fibrosus was determined with gagCEST in non-degenerated discs according to Pfirrmann. ER were examined during the peak of their competition preparation (T0) and 6 months later during the peak of their post-competition recovery period ( $\mathrm{T} 1$ ).

Results: At TO we found significantly higher gagCEST values in ER (A) compared to HV (C) (NP: $4.26 \pm 2.37 \%$ vs. $3.38 \pm 1.72 \%, p<0.05$; confidence interval (Cl) $0.32 \% / 1.44 \%$; AF: $2.75 \pm 1.7 \%$ vs. $1.961 \pm 1.23 \%, \mathrm{p}<0.01$; Cl 0.4\%/1.2\%). At
T1 gagCEST values in ER (B) decreased and illustrated no significant difference compared to HV (C) (NP: $3.55 \pm 2.31 \%, p=0.531, \mathrm{Cl} 0.038 \% / 0.73 \%$; AF: 2.31 $\pm 1.57 \%, p=0.073, \mathrm{Cl} 0.03 \% / 0.74 \%$ ).

Conclusion: Compared to HV lumbar IVD of ER show significantly higher gagCEST values during the peak of their competition preparation and similar values during the recovery period, indicating a GAG remodelling effect by training.

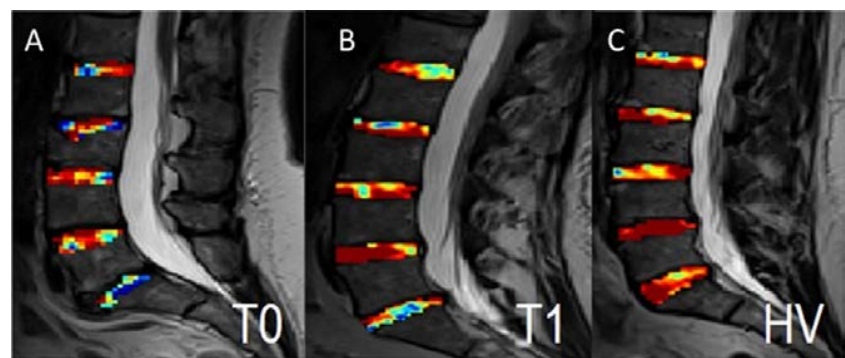

Figure 1. Comparison of gagCEST values of lumbar IVD between ER (A, TO; B, T1) and HV

Disclosure of Interests: : Philipp Sewerin Grant/research support from: AbbVie Deutschland $\mathrm{GmbH}$ \& Co. KG

Bristol-Myers Squibb Celgene $\mathrm{GmbH}$

Lilly Deutschland $\mathrm{GmbH}$

Novartis Pharma GmbH Pfizer Deutschland GmbH

Rheumazentrum Rhein-Ruhr, Consultant of:AMGEN GmbH AbbVie Deutschland $\mathrm{GmbH} \&$ Co. KG Biogen GmbHBristol-Myers Squibb Celgene $\mathrm{GmbH}$ Chugai Pharma arketing Ltd. / Chugai Europe GmbHHexal Pharma Janssen-Cilag GmbH Johnson \& Johnson Deutschland GmbHLilly Deutschland GmbH / Lilly Europe / Lilly Global Novartis Pharma GmbH Pfizer Deutschland GmbH Roche Pharma Rheumazentrum Rhein-Ruhr Sanofi-Genzyme Deutschland $\mathrm{GmbH}$ Swedish Orphan Biovitrum GmbH UCB Pharma GmbH, Speakers bureau: AMGEN GmbH AbbVie Deutschland GmbH \& Co. KG Biogen GmbHBristol-My ers Squibb Celgene GmbH Chugai Pharma arketing Ltd. / Chugai Europe GmbH Hexal Pharma Janssen-CilagGmbH Johnson \& Johnson Deutschland GmbHLilly Deutschland GmbH / Lilly Europe / Lilly Global Novartis Pharma GmbH Pfizer Deutschland $\mathrm{GmbH}$ Roche Pharma Rheumazentrum Rhein-Ruhr Sanofi-Genzyme Deutschland $\mathrm{GmbH}$ Swedish Orphan Biovitrum GmbH UCB Pharma $\mathrm{GmbH}$, Daniel Abrar: None declared, Miriam Frenken: None declared, Matthias Schneider Grant/research support from: GSK, UCB, Abbvie, Consultant of: Abbvie, Alexion, Astra Zeneca, BMS, Boehringer Ingelheim, Gilead, Lilly, Sanofi, UCB, Speakers bureau: Abbvie, Astra Zeneca, BMS, Chugai, GSK, Lilly, Pfizer Sanofi, Benedikt Ostendorf: None declared, Christoph Schleich: None declared DOI: 10.1136/annrheumdis-2020-eular.6453

THURSDAY, 04 JUNE 2020

Pain in rheumatic diseases, including fibromyalgia _

THU0477 SURAL NERVE SIZE IN FIBROMYALGIA SYNDROME

Figure 2. Prevalence ad distribution of sing/symptoms indicative for the presence of smallfiber pathology

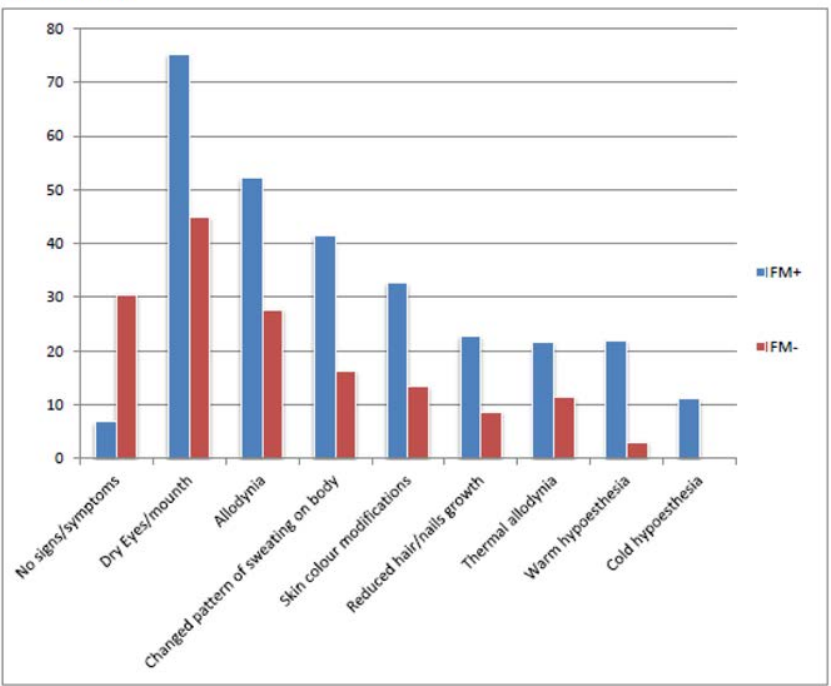

Values reported in percentage. All between-groups differences were statistically significant $(p<0.01)$ 